\title{
Reversible quantum teleportation in an optical lattice
}

\author{
Luis Santos and Dagmar Bruß \\ Institut für Theoretische Physik, Universität Hannover, Appelstr. 2, D-30167 Hannover, Germany
}

We propose a protocol, based on entanglement procedures recently suggested by [D. Jaksch et al., Phys. Rev. Lett. 82, 1975 (1999)], which allows the teleportation of an unknown state of a neutral atom in an optical lattice to another atom in another site of the lattice, without any irreversible detection.

\section{INTRODUCTION}

The characterization, creation, control and manipulation of quantum entanglement [1,2] constitutes the basis of the fast-developing research field of quantum information. An entangled state of two or more [3] particles can be intuitively understood as the situation in which the state of one particle cannot be determined independently from the state of the others. Of course, the concept deserves more technical definitions and quantifications, but these are beyond the scope of this paper.

Quantum computing [4], quantum cryptography [5], and other interesting phenomena, constitute excellent examples of the extraordinary potential of quantum entanglement. Among these phenomena, quantum teleportation [6] is perhaps one of the most striking ones. Quantum teleportation consists in the transport of a quantum state from one particle to another in a disembodied way. Following the theoretical proposal of Bennett et al [6], quantum teleportation has been recently demonstrated experimentally [7]. This teleportation scheme involves a Bell-measurement [8], and therefore it is performed in an irreversible way. However, it has been shown [9, 10 that quantum teleportation can be performed in a reversible way, i.e. without any irreversible detection. In order to obtain such unitary teleportation it is necessary to consider the detector as a quantum mechanical object, the state of which is not read out. In this paper, we show how this idea can be implemented in a particular physical situation.

Several physical systems have been proposed, in which entanglement can be created and manipulated, as for example cavity QED [11], photons [12] and ion-traps [13. Recently it has been proposed that neutral atoms in an optical lattice can be entangled in a controlled way by using cold collisions between them [14]. It has been shown [15] how one can use this entanglement mechanism to create GHZ-states [3], or implement parallel quantum computing and quantum error correction 16. We show in the present paper, that in the framework of this particular entanglement procedure it is possible to implement a reversible teleportation protocol.

The structure of the paper is as follows. In Sec. II we review the irreversible and reversible teleportation schemes, and also present the abstract formulation of our teleportation proposal. In Sec. III, we briefly review the entanglement procedure of [14,15]. In Sec. IV, we consider explicitly the most simple case of teleportation with just three lattice sites, whereas in Sec. V we analyze the case of an arbitrary number of sites. We finalize in Sec. VI with some conclusions.

\section{TELEPORTATION PROTOCOLS}

In this section, we review briefly the general ideas behind the teleportation schemes both with and without irreversible measurements. For a more detailed discussion we refer to Refs. 66,9, 10]. At the end of the section we present the abstract formalism of our teleportation scheme. In this section, we follow the notation of Ref. 9.

\section{A. Irreversible teleportation}

We are considering three two-level systems, denoted by index 1,2 and 3 , each with basis states $\{|0\rangle,|1\rangle\}$. Initially particle 1 is in an unknown state $|\phi\rangle$, whereas particles 2 and 3 are in a maximally entangled state $\left|\phi^{+}\right\rangle=(|0\rangle|0\rangle+|1\rangle|1\rangle) / \sqrt{2}$. Particles 1 and 2 are together at one place, and particle three is at a different place. The teleportation scheme can be well understood using the decomposition [6]:

$$
\left|\phi_{\text {in }}\right\rangle=|\phi\rangle_{1}\left|\phi^{+}\right\rangle_{23}=\frac{1}{2} \sum_{J=0}^{3}\left|\psi^{(J)}\right\rangle_{12} U_{3}^{(J) \dagger}|\phi\rangle_{3},
$$

where $\left|\psi^{(J)}\right\rangle$ is the entangled basis for two qubits,

$$
\begin{aligned}
\left|\psi^{(J)}\right\rangle & =\sum_{l=0}^{1} \exp (\pi i l n)|l\rangle|(l+m) \bmod 2\rangle / \sqrt{2} \\
U^{(J)} & =\sum_{k=0}^{1} \exp (\pi i k n)|k\rangle\langle(k+m) \bmod 2|
\end{aligned}
$$

with

$$
J=n \cdot 2+m \quad, \quad \text { i.e. } n=J \operatorname{div} 2 ; m=J \bmod 2 \text {. }
$$

Explicitly this corresponds to $\left|\psi^{(0)}\right\rangle \equiv\left|\phi^{+}\right\rangle,\left|\psi^{(1)}\right\rangle \equiv$ $\left|\psi^{+}\right\rangle,\left|\psi^{(2)}\right\rangle \equiv\left|\phi^{-}\right\rangle,\left|\psi^{(3)}\right\rangle \equiv\left|\psi^{-}\right\rangle$, the well-known Bell 
states [8], and $U^{(0)} \equiv \mathbb{1}, U^{(1)} \equiv \sigma_{1}, U^{(2)} \equiv \sigma_{3}, U^{(3)} \equiv$ $i \sigma_{2}$, where $\sigma_{j}$ are the Pauli matrices.

The teleportation scheme [6] works as follows: first a joint measurement of the state of particles 1 and 2, i.e. a Bell measurement, is performed; then, using a classical channel, the result $J$ of the measurement is sent to the other site and, using the value $J$, the appropriate unitary transformation $U_{3}^{(J)}$ is performed on particle 3 to transform the state of particle 3 into $|\phi\rangle$. As we observe, this procedure is clearly irreversible, because a measurement of the joint state of 1 and 2 is necessary.

In the following a lower index $1,2,3, \ldots$ for a unitary transformation refers to the particle to which the transformation is applied, and a lower index $a, b, c$ refers to the sequence of transformations.

\section{B. Reversible teleportation}

However, the previous scheme is not the only one which allows to teletransport an unknown state. In particular, there is a reversible way to perform such a task [9, 10]. Let us assume an auxiliary particle $A$ (which in the following we shall call ancilla), which is a four-level system with basis states $\{|0\rangle,|1\rangle,|2\rangle,|3\rangle\}$. The initial state of the system (11) becomes now:

$$
\left|\phi_{i n}\right\rangle=|0\rangle_{A}|\phi\rangle_{1}\left|\phi^{+}\right\rangle_{23}
$$

The reversible teleportation scheme works as follows. First, we perform the following unitary operation on the initial state:

$$
U_{a}=\sum_{J=0}^{3}\left|\psi^{(J)}\right\rangle_{12} O_{A}^{(J)}\left\langle\left.\psi^{(J)}\right|_{12},\right.
$$

where $O_{A}^{(0)} \equiv \mathbb{1}$ and $O_{A}^{(J)} \equiv|0\rangle\langle J|+| J\rangle\left\langle 0\left|+\sum_{k \neq 0, J}^{3}\right| k\right\rangle\langle k|$, for $0<J \leq 3$. It is easy to observe that the state of the system becomes:

$$
U_{a}\left|\phi_{i n}\right\rangle=\frac{1}{2} \sum_{J=0}^{3}|J\rangle_{A}\left|\psi^{(J)}\right\rangle_{12} U_{3}^{(J) \dagger}|\phi\rangle_{3} .
$$

A second step consists in transporting the particle $A$ near particle 3 , and performing a unitary transformation of the form:

$$
U_{b}=\sum_{J=0}^{3}|J\rangle_{A} U_{3}^{(J)}\left\langle\left. J\right|_{A}\right.
$$

i.e. a unitary transformation on 3 conditional to the value of the state of the ancilla $A$. After performing operation $U_{b}$ the system becomes

$$
U_{b} U_{a}\left|\phi_{i n}\right\rangle=\frac{1}{2}|\phi\rangle_{3} \sum_{J=0}^{3}|J\rangle_{A}\left|\psi^{(J)}\right\rangle_{12}
$$

and therefore the state $|\phi\rangle$ has been transported into 3, whatever the final value of 1,2 and $A$. Eventually, if $A$ were transported back near 1 and 2 , one could perform a unitary operation to restore 1,2 and $A$ to their original states.

\section{Our teleportation scheme}

In this section, we present the general idea behind our reversible teleportation scheme, in order to observe the similarities and differences in comparison with the scheme presented previously. In the following we consider three two-level systems $1,2,3$. Note that, as we show in Sec. $\mathrm{V}$, the method can be generalized to an arbitrary number of particles. The lowest non-trivial number of particles is three and therefore used to explain our scheme. Due to the restrictions of the physical model that we employ in Sec. III, all particles, including the ancillas, are in our case qubits, i.e. have just two states $\{|0\rangle,|1\rangle\}$ (in the case of the ancillas we shall consider a different state $|2\rangle$ instead of $|1\rangle)$. In order to perform the teleportation, though, we need two bits of information and therefore two ancillas $A_{1}$ and $A_{2}$. We assume that the initial state of the system is of the form

$$
\left|\phi_{i n}\right\rangle=|0\rangle_{A_{1}}|0\rangle_{A_{2}}|\phi\rangle_{1}|0\rangle_{2}|0\rangle_{3}
$$

As one can observe, this is a difference with respect to the initial state considered in expression (1): the particles 2 and 3 are not initially entangled, and as we will see later they will become entangled with each other as well as with particle 1 during our transformations.

The first step of our teleportation scheme consists in performing a unitary transformation $V_{a}$ acting on the 6dimensional space of the three particles 1,2 and 3 , in such a way that the state of the system becomes

$$
V_{a}\left|\phi_{i n}\right\rangle=\sum_{J=0}^{3}|0\rangle_{A_{1}}|0\rangle_{A_{2}}\left|\tilde{\psi}^{(J)}\right\rangle_{12} \tilde{U}_{3}^{(J) \dagger}|\phi\rangle_{3}
$$

where the exact definitions of $\left|\tilde{\psi}^{(J)}\right\rangle_{12}, \tilde{U}_{3}^{(J)}$, and $V_{a}$ will be presented in Sec. IV. As we observe, the operation $V_{a}$ entangles the particles 1, 2 and 3 . The next step of the teleportation scheme is to perform a unitary operation of the form:

$$
V_{b} \equiv \sum_{J=0}^{3}\left|\tilde{\psi}^{(J)}\right\rangle_{12} O_{A_{1} A_{2}}^{(J)}\left\langle\left.\tilde{\psi}^{(J)}\right|_{12}\right.
$$

where $O_{A_{1} A_{2}}^{(J)}=\sigma_{1, A_{1}}^{J \bmod 2} \sigma_{1, A_{2}}^{J \operatorname{div} 2}$ (our notation is $\sigma_{i}^{0}=\mathbb{1}$ and $\left.\sigma_{i}^{1}=\sigma_{i}\right)$. After applying $V_{b}$ the state of the system takes the form

$$
V_{b} V_{a}\left|\phi_{i n}\right\rangle=\sum_{J=0}^{3}\left|\tilde{\psi}^{\prime(J)}\right\rangle_{A_{1} A_{2}}\left|\tilde{\psi}^{(J)}\right\rangle_{12} U_{3}^{(J) \dagger}|\phi\rangle_{3}
$$


where the exact form of $\left|\tilde{\psi}^{\prime(J)}\right\rangle_{A_{1} A_{2}}$ is presented in Sec. IV. This step can be called the "reading of the states by the ancillas". Once this is done, we perform a unitary operation of the form:

$$
V_{c} \equiv \sum_{J=0}^{3}\left|\tilde{\psi}^{\prime(J)}\right\rangle_{A_{1} A_{2}} R_{3}^{(J)}\left\langle\left.\tilde{\psi}^{\prime(J)}\right|_{A_{1} A_{2}}\right.
$$

where $R_{3}^{(J)} \tilde{U}_{3}^{(J) \dagger}=c_{J} \tilde{U}_{3}^{(0) \dagger}$. Here the coefficients $c_{J}$ can be \pm 1 , and are in detail calculated in Sec. IV. Then, the state of the system becomes

$$
V_{c} V_{b} V_{a}\left|\phi_{i n}\right\rangle=\tilde{U}_{3}^{(0) \dagger}|\phi\rangle_{3} \otimes \sum_{J=0}^{3} c_{J}\left|\tilde{\psi}^{\prime(J)}\right\rangle_{A_{1} A_{2}}\left|\tilde{\psi}^{(J)}\right\rangle_{12},
$$

where we have moved the state of 3 out of the sum to stress that it is now independent of the state of 1,2 and the ancillas. As a final step, we just need to perform the unitary operation $\tilde{U}_{3}^{(0)}$ to conclude the teleportation. The ancillas and 1 and 2 remain in an entangled state. Finally, but this is not necessary for the teleportation, if we perform again $V_{b}$ the ancillas are brought back to their original state $|0\rangle_{A_{1}}|0\rangle_{A_{2}}$.

\section{QUANTUM ENTANGLEMENT OF ATOMS IN OPTICAL LATTICES}

In this section we present the physical system in which we shall implement our reversible teleportation scheme, briefly reviewing Refs. 14,15]. Let us consider a collection of bosonic neutral atoms occupying the sites of an optical lattice. In order to perform the necessary quantum logical operations one has to be able to fill the lattice wells with exactly one particle each. This can be achieved - at present only theoretically - by loading the lattice from a Bose-Einstein Condensate (BEC), and inducing at sufficiently low temperatures a phase transition from the superfluid BEC phase into a Mott insulator phase, by increasing the ratio between the interaction energy inside each well and the tunneling rate between the wells, as predicted by the Bose-Hubbard model [17]. In addition to single-atom operations [18] two basic two-atom operations can be perfomed within this physical scheme, based on cold collisions between the atoms.

\section{A. Shift operation}

The two internal states of the atoms carrying the quantum information are called $\{|0\rangle,|1\rangle\}$. As shown in detail in Ref. 15], by properly arranging the detuning and polarization of the lasers which form the lattice, it can be achieved that each of the internal atomic levels can see a different potential. In particular, by changing the dephasing between the circularly polarized waves $\sigma^{ \pm}$which form the lattice, the potentials for the different internal levels move in opposite directions. Let us suppose that the lattice of $|0\rangle$ moves to the right, while the lattice of $|1\rangle$ moves to the left. Two neighbour atoms in the lattice occupy sites $j$ and $j+1$ (our numbering is from left to right). It is clear that, using a lattice displacement, the neighbour atoms can only undergo a collision if the atom at $j$ is in $|0\rangle$ and the one at $j+1$ is in $|1\rangle$. Any other situation prevents the atoms from approaching each other. When the two particles are put in contact, they can interact via s-wave scattering. As a result, a collisional phase appears, which can be controlled by basically changing the interaction time. In particular, we shall choose this collisional phase to be $\pi$. After the desired time the lattice is brought back to its original position [19]. We shall call this operation shift operation, following the notation of [15], and it can be summarized in the following table:

$$
\begin{aligned}
|0\rangle_{j}|0\rangle_{j+1} & \rightarrow|0\rangle_{j}|0\rangle_{j+1}, \\
|0\rangle_{j}|1\rangle_{j+1} & \rightarrow-|0\rangle_{j}|1\rangle_{j+1}, \\
|1\rangle_{j}|0\rangle_{j+1} & \rightarrow|1\rangle_{j}|0\rangle_{j+1}, \\
|1\rangle_{j}|1\rangle_{j+1} & \rightarrow|1\rangle_{j}|1\rangle_{j+1} .
\end{aligned}
$$

\section{B. Sweep operation}

Let us assume that the atoms have a third atomic level $|2\rangle$, which can be displaced like the levels $|0\rangle$ and $|1\rangle$ by using the corresponding transport lattice. The operation is basically like the previous one, but now only those atoms in level $|2\rangle$ are going to participate. In particular, we are going to consider that just the ancilla is excited into the level $|2\rangle$. The interaction of the ancilla with an atom in the site $j$ can be designed (following the same arguments as above) in such a way that [20]:

$$
\begin{array}{r}
|2\rangle_{A}|0\rangle_{j} \rightarrow|2\rangle_{A}|0\rangle_{j} \\
|2\rangle_{A}|1\rangle_{j} \rightarrow-|2\rangle_{A}|1\rangle_{j}
\end{array}
$$

Following [15], we shall call this operation sweep operation. By varying the speed by which the lattice of the state $|2\rangle$ is moved during the sweep operation, it is possible to act on a particular site of the lattice, even when the ancilla crosses through other sites in the lattice, in particular because the collisional time can be designed in such a way that for undesired sites the collisional phase is a multiple of $2 \pi$ [21].

\section{REVERSIBLE TELEPORTATION PROTOCOL: THREE SITES ONLY}

Let us now show explicitly the transformations for our scheme of reversible teleportation. In this section we explain the most simple case, in which we have three sites, 
each one occupied by one atom. We shall call these atoms (from left to right in the lattice) 1,2 and 3. We shall consider another two atoms $A_{1}$ and $A_{2}$, which will act as ancillas, and which are initially placed sufficiently apart (at the left) from the sites 1,2 and 3; this requirement is necessary to avoid that the unitary operations applied on the site 1, 2 and 3 could affect the ancillas, and vice versa. We shall also consider that both ancillas are separated by a distance larger than the dimensions of the three sites 1, 2 and 3 ; this requirement is necessary to avoid that during the operation of one ancilla on the sites, the other sites could be affected by the other ancilla. We shall discuss in Sec. VI the case in which only one ancilla is present. We begin with the initial state of the system:

$$
\left|\phi_{\text {in }}\right\rangle=|0\rangle_{A_{1}}|0\rangle_{A_{2}}|\phi\rangle_{1}|0\rangle_{2}|0\rangle_{3},
$$

where $|\phi\rangle=a|0\rangle+b|1\rangle$. Our objective is to transport this state from particle 1 to particle 3 , in a reversible way, using the operations of Sec. III. Our teleportation scheme consists on the following three general steps:

\section{A. Creation of the entanglement between 1,2 and 3}

As a first step, we perform a Hadamard transform $(H)$

$$
\begin{aligned}
& |0\rangle \rightarrow \frac{1}{\sqrt{2}}(|0\rangle+|1\rangle), \\
& |1\rangle \rightarrow \frac{1}{\sqrt{2}}(|0\rangle-|1\rangle),
\end{aligned}
$$

in each one of the sites 1,2 and 3 [18]. Then, we perform a shift operation (of the lattices of $|0\rangle$ and $|1\rangle$ ), and after that we perform another Hadamard transformation to all the sites. As a result of these three operations the state of the system becomes:

$$
V_{a}\left|\phi_{i n}\right\rangle=\frac{1}{2}|0\rangle_{A_{1}}|0\rangle_{A_{2}} \sum_{J=0}^{3}\left|\tilde{\psi}^{(J)}\right\rangle_{12} \tilde{U}_{3}^{(J) \dagger}|\phi\rangle_{3},
$$

where $\left|\tilde{\psi}^{(J)}\right\rangle_{12}=|J \operatorname{div} 2\rangle_{1}|J \bmod 2\rangle_{2}$, and $\tilde{U}^{(0)}=-i \sigma_{2}$, $\tilde{U}^{(1)}=\sigma_{1}, \tilde{U}^{(2)}=-\sigma_{3}, \tilde{U}^{(3)}=\mathbb{1}$. As we observe the state of the three sites becomes now entangled.

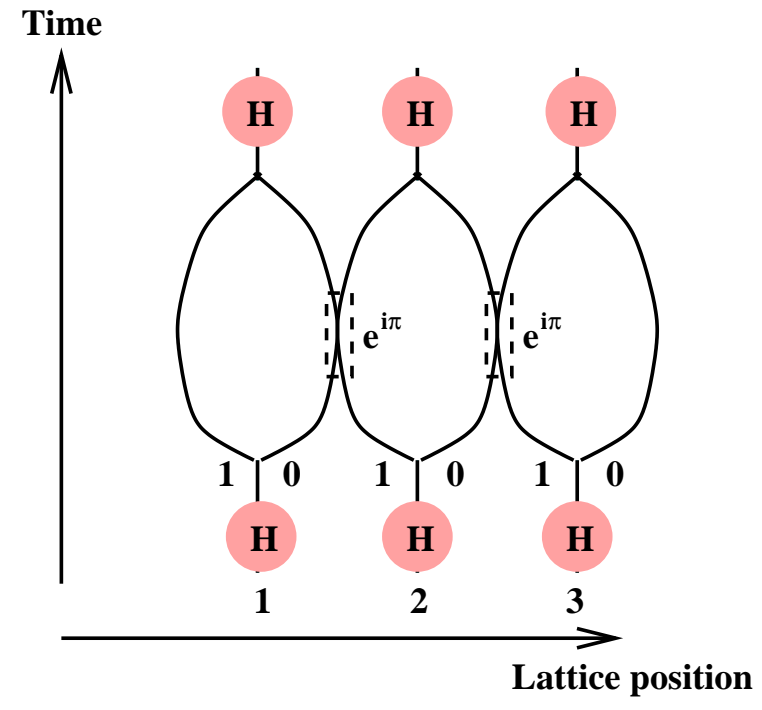

FIG. 1. Creation of the entanglement between sites 1,2 and 3. We have depicted the shift operation as a multiparticle interferometer following Ref. [15]. Here $H$ denotes a Hadamard transform.

\section{B. Reading of 1 and 2 using the ancillas}

First, a Hadamard transform is performed in each one of the ancillas (between levels $|0\rangle$ and $|2\rangle$ ):

$$
\begin{aligned}
H_{A} V_{a}\left|\phi_{i n}\right\rangle= & \frac{1}{4}\left(|0\rangle_{A_{1}}+|2\rangle_{A_{1}}\right)\left(|0\rangle_{A_{2}}+|2\rangle_{A_{2}}\right) \\
& \otimes \sum_{J=0}^{3}\left|\tilde{\psi}^{(J)}\right\rangle_{12} \tilde{U}_{3}^{(J) \dagger}|\phi\rangle_{3} .
\end{aligned}
$$

Then, we perform a sweep to the right of the lattice of $|2\rangle$ until placing the ancilla $A_{1}$ in site 2 , and after that sweeping again to the right we place the ancilla $A_{2}$ in site 1 . In both cases, the interaction times are properly designed to obtain a sweep operation as described in (16). Finally we displace the lattice of $|2\rangle$ back to its original position. The state of the system after this operation $\tilde{V}_{b}$ becomes:

$$
\begin{aligned}
\tilde{V}_{b} H_{A} V_{a}\left|\phi_{i n}\right\rangle= & \frac{1}{4} \sum_{J=0}^{3}\left(|0\rangle_{A_{1}}+(-1)^{J \bmod 2}|2\rangle_{A_{1}}\right) \\
& \left(|0\rangle_{A_{2}}+(-1)^{J \operatorname{div} 2}|2\rangle_{A_{2}}\right) \\
& \otimes\left|\tilde{\psi}^{(J)}\right\rangle_{12} \tilde{U}_{3}^{(J) \dagger}|\phi\rangle_{3} .
\end{aligned}
$$




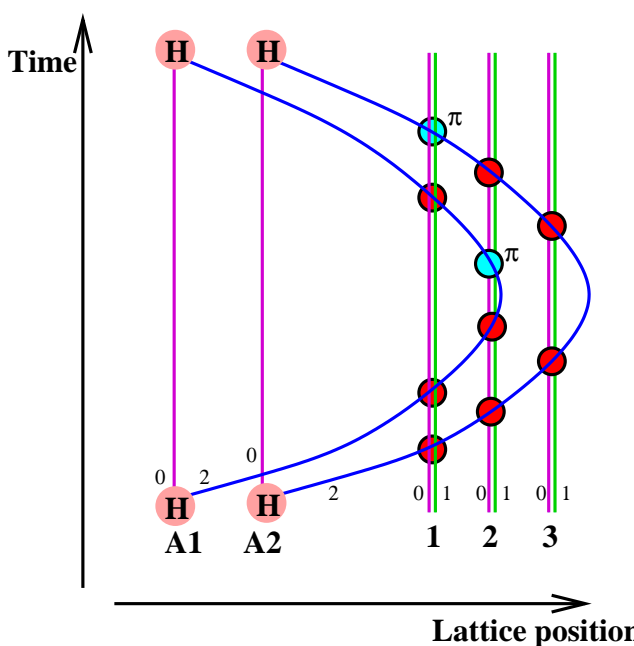

FIG. 2. Reading of the state of the sites 1 and 2 using the ancillas $A_{1}$ and $A_{2} . H$ denotes a Hadamard transform, and the circles sweep operations. The sweep operations which introduce a phase $\pi$ as in $(16)$ are indicated. The rest are assumed to lead to a zero phase.

Performing a new Hadamard transform in both ancillas, the state of the system becomes:

$$
V_{b} V_{a}\left|\phi_{i n}\right\rangle=\frac{1}{2} \sum_{J=0}^{3}\left|\tilde{\psi}^{\prime(J)}\right\rangle_{A_{1} A_{2}}\left|\tilde{\psi}^{(J)}\right\rangle_{12} \tilde{U}_{3}^{(J) \dagger}|\phi\rangle_{3},
$$

where $\left|\tilde{\psi}^{\prime(J)}\right\rangle_{A_{1} A_{2}}=|J \bmod 2\rangle_{A_{1}}|J \operatorname{div} 2\rangle_{A_{2}}$ and $V_{b}=$ $H_{A} \tilde{V}_{b} H_{A}$. Therefore, we have copied the state of 1 (2) into $A_{2}\left(A_{1}\right)$. Note that the joint operation $V_{b}$ is equivalent to a sequence of two CNOT gates (i) with 2 as control qubit and $A_{1}$ as target; (ii) with 1 as control qubit and $A_{2}$ as target.

\section{Teleportation}

Now, we are going to use the values of the ancillas to teleport the state $|\phi\rangle$ into the site 3 . First of all, we perform a Hadamard transform in the site 3 (in principle this operation can be performed simultaneously in the other two sites [18], but since the other sites remain untouched during this step, we just consider for simplicity that only the site 3 is affected by this operation). Then, the lattice of $|2\rangle$ is swept to the right until $|2\rangle_{A_{2}}$ is in contact with the site 3 and interacts following the rule of (16). Let us call this operation $\tilde{V}_{c}$. After that we perform again a Hadamard transform in the site 3 . One calculates that the effect of these steps is to change the state of the system into

$$
\begin{aligned}
& \frac{1}{2}\left(\left|\tilde{\psi}^{\prime(0)}\right\rangle_{A_{1} A_{2}}\left|\tilde{\psi}^{(0)}\right\rangle_{12}-\left|\tilde{\psi}^{\prime(2)}\right\rangle_{A_{1} A_{2}}\left|\tilde{\psi}^{(2)}\right\rangle_{12}\right) \tilde{U}_{3}^{(0) \dagger}|\phi\rangle_{3} \\
& +\frac{1}{2}\left(\left|\tilde{\psi}^{\prime(1)}\right\rangle_{A_{1} A_{2}}\left|\tilde{\psi}^{(1)}\right\rangle_{12}+\left|\tilde{\psi}^{\prime(3)}\right\rangle_{A_{1} A_{2}}\left|\tilde{\psi}^{(3)}\right\rangle_{12}\right) \tilde{U}_{3}^{(1) \dagger}|\phi\rangle_{3}
\end{aligned}
$$

\section{REVERSIBLE TELEPORTATION PROTOCOL: GENERALIZATION TO ARBITRARY NUMBER OF SITES}

In this section we shall show how our teleportation scheme can be extended to the case in which we have $N$ sites, instead of 3 as in Sec. IV. We shall consider that $N$ is an even number, i.e. $N=2 m$, but similar procedures can be designed for odd $N$ (as we have already shown for 
the case of $N=3$ in Sec. [V] . Therefore, our physical system is now composed of $N$ two-level atoms each in one site of the lattice, and 2 ancillas. We consider that the initial state of the system is of the form:

$$
\left|\phi_{i n}\right\rangle=|0\rangle_{A_{1}}|0\rangle_{A_{2}}|\phi\rangle_{1} \bigotimes_{j=2}^{N}|0\rangle_{j}
$$

\section{A. Creation of the entanglement between $1,2 \ldots, N$}

As for the case of 3 sites, we perform as a first step a Hadamard transform in the each one of the sites $1, \cdots, N$. Then, we perform a shift operation (of the lattices of $|0\rangle$ and $|1\rangle$ ), and after that we perform another Hadamard transformation on all the sites. In Appendix A, we demonstrate that after applying the previous three operations the state of the system becomes (remember that $m=N / 2)$ :

$$
V_{a}\left|\phi_{i n}\right\rangle=\frac{1}{2^{m}}|0\rangle_{A_{1}}|0\rangle_{A_{2}} \sum_{J=0}^{2^{N}-1} c_{J}\left|\tilde{\psi}^{(J)}\right\rangle_{1 \cdots N-1} \tilde{U}_{N}^{(J) \dagger}|\phi\rangle_{N},
$$

where

$$
\left|\tilde{\psi}^{(J)}\right\rangle_{1 \cdots N-1}=\bigotimes_{k=1}^{N-1}\left|a_{k}^{(J)}\right\rangle_{k}
$$

with $J=\sum_{k=1}^{N-1} a_{k}^{(J)} 2^{N-1-k}$, and $c_{J}$ can be \pm 1 . We define:

$$
\begin{array}{r}
S_{e}^{(J)}=\sum_{k=1}^{m-1} a_{2 k}^{(J)}, \\
S_{o}^{(J)}=\sum_{k=1}^{m} a_{2 k-1}^{(J)},
\end{array}
$$

which count the number of $|1\rangle$ 's in the even sites (except $N$ ) and in the odd sites, respectively. We show in App. A, that the following holds if $m$ is an even number:

- if $S_{e}^{(J)} \bmod 2=0, S_{o}^{(J)} \bmod 2=0$, then $\tilde{U}_{N}^{(J)}=W^{(0)} \equiv 1+i \sigma_{2}$.

- if $S_{e}^{(J)} \bmod 2=1, S_{o}^{(J)} \bmod 2=0$, then $\tilde{U}_{N}^{(J)}=W^{(1)} \equiv 1-i \sigma_{2}$.

- if $S_{e}^{(J)} \bmod 2=0, S_{o}^{(J)} \bmod 2=1$, then $\tilde{U}_{N}^{(J)}=W^{(2)} \equiv \sigma_{3}+\sigma_{1}$.

- if $S_{e}^{(J)} \bmod 2=1, S_{o}^{(J)} \bmod 2=1$, then $\tilde{U}_{N}^{(J)}=W^{(3)} \equiv \sigma_{3}-\sigma_{1}$.

If $m$ is odd the same is valid but one has to interchange $S_{e, o}^{(J)} \bmod 2=0 \leftrightarrow S_{e, o}^{(J)} \bmod 2=1$.

\section{B. Reading of $1, \cdots, N-1$ using the ancillas}

As for the case of just 3 sites, we first perform a Hadamard transform in each one of the ancillas (between levels $|0\rangle$ and $|2\rangle)$ :

$$
\begin{aligned}
H_{A} V_{a}\left|\phi_{i n}\right\rangle= & \frac{1}{2^{m+1}}\left(|0\rangle_{A_{1}}+|2\rangle_{A_{1}}\right)\left(|0\rangle_{A_{2}}+|2\rangle_{A_{2}}\right) \\
& \otimes \sum_{J=0}^{2^{N}-1} c_{J}\left|\tilde{\psi}^{(J)}\right\rangle_{1 \cdots N-1} \tilde{U}_{N}^{(J) \dagger}|\phi\rangle_{N} .
\end{aligned}
$$

Then, we perform a sweep to the right of the lattice of $|2\rangle$ until placing the ancilla $|2\rangle_{A_{1}}$ in site $N-1$. Then, we displace $|2\rangle_{A_{1}}$ to the left, in such a way that a sweep operation (16) is performed every 2 sites beginning in $N-1$, i.e. in the sites $N-1, N-3, \cdots, 3,1$. After that we sweep back to the right the ancilla state $|2\rangle_{A_{2}}$, in such a way that a sweep operation (16) is performed every 2 sites beginning in $N-2$, i.e. in the sites $N-2, N-4, \cdots, 4,2$. Finally we displace the lattice of $|2\rangle$ back to its original position. The state of the system becomes:

$$
\begin{aligned}
& \tilde{V}_{b} H_{A} V_{a}\left|\phi_{i n}\right\rangle=\frac{1}{2^{m+1}} \sum_{J=0}^{2^{N}-1} c_{J}\left(|0\rangle_{A_{1}}+(-1)^{S_{e}^{(J)} \bmod 2}|2\rangle_{A_{1}}\right) \\
& \left(|0\rangle_{A_{2}}+(-1)^{S_{o}^{(J)} \bmod 2}|2\rangle_{A_{2}}\right) \otimes\left|\tilde{\psi}^{(J)}\right\rangle_{1 \cdots N-1} \tilde{U}_{N}^{(J) \dagger}|\phi\rangle_{N} \cdot
\end{aligned}
$$

Performing a new Hadamard transform in both ancillas, the state of the system becomes:

$$
\begin{aligned}
& V_{b} V_{a}\left|\phi_{i n}\right\rangle=\frac{1}{2^{m}} \sum_{J=0}^{2^{N}-1} c_{J}\left|S_{e}^{(J)} \bmod 2\right\rangle_{A_{1}}\left|S_{o}^{(J)} \bmod 2\right\rangle_{A_{2}} \\
& \left|\tilde{\psi}^{(J)}\right\rangle_{1 \cdots N-1} \tilde{U}_{N}^{(J) \dagger}|\phi\rangle_{N}
\end{aligned}
$$

with $V_{b}=H_{A} \tilde{V}_{b} H_{A}$.

\section{Teleportation}

As in Sec. [IV, we are going to use the values of the ancillas to teleport the state $|\phi\rangle$ into the site $N$. First we perform a Hadamard transform in the site $N$. Then, the lattice of $|2\rangle$ is swept to the right until $|2\rangle_{A_{2}}$ is in contact with the site $N$ and interacts following the rule of (16). Let us call this operation $\tilde{V}_{c}$. After that we perform again a Hadamard transform in the site $N$. The effect of these steps is to change the state of the system into

$$
\begin{aligned}
& \frac{1}{2^{m}} \sum_{J=0, S_{o}^{(J)} \bmod 2=0}^{2^{N}-1} c_{J}^{\prime}\left|S_{e}^{(J)} \bmod 2\right\rangle_{A_{1}}|0\rangle_{A_{2}} \\
& \otimes\left|\tilde{\psi}^{(J)}\right\rangle_{1 \cdots N-1} W_{N}^{(0) \dagger}|\phi\rangle_{N} \\
& +\frac{1}{2^{m}} \sum_{J=0, S_{o}^{(J)} \bmod 2=1}^{2^{N}-1} c_{J}^{\prime}\left|S_{e}^{(J)} \bmod 2\right\rangle_{A_{1}}|1\rangle_{A_{2}} \\
& \left|\tilde{\psi}^{(J)}\right\rangle_{1 \cdots N-1} W_{N}^{(1) \dagger}|\phi\rangle_{N},
\end{aligned}
$$


where $c_{J}^{\prime}$ can be \pm 1 . After this, we move further to the right the lattice of $|2\rangle$ until $|2\rangle_{A_{1}}$ enters in contact with the site $N$, interacts with it following the rule of (16), and we sweep back the lattice of $|2\rangle$ to its original position. Let us call this operation $\tilde{V}_{c}^{\prime}$. After the joint operation $V_{c}=\tilde{V}_{c}^{\prime} H_{A} \tilde{V}_{c} H_{A}$, the state of the system takes the form:

$V_{c} V_{b} V_{a}\left|\phi_{i n}\right\rangle=$

$\frac{1}{2^{m}}\left[\sum_{J=0}^{2^{N}-1} c_{J}^{\prime \prime}\left|S_{e}^{(J)} \bmod 2\right\rangle_{A_{1}}\left|S_{o}^{(J)} \bmod 2\right\rangle_{A_{2}}\left|\tilde{\psi}^{(J)}\right\rangle_{1 \cdots N-1}\right]$

$\otimes W_{N}^{(0) \dagger}|\phi\rangle_{N}$.

where again $c_{J}^{\prime \prime}$ can be \pm 1 . Then, we just need to perform the unitary operation $W_{N}^{(0)}$ to complete the teleportation. As in Sec. IV, if we perform again the operations of reading of the ancilla, it is possible to bring back the ancillas to their initial value $|0\rangle_{A_{1}}|0\rangle_{A_{2}}$, but as previously this is not necessary for the teleportation.

\section{CONCLUSIONS}

In this paper we have presented a teleportation scheme which allows to teleport an atomic state of a two-level atom confined in some site of an optical lattice to another two-level atom in a distant site of the lattice, in a reversible way. In order to achieve that, we have used entanglement procedures recently developped in Refs. 114,15. We have shown the similarities and differences of our teleportation scheme in comparison with other reversible teleportation schemes. A difference is that we begin the teleportation process with a disentangled system, and perform a shift operation which entangles all the sites of the lattice; in particular the particle which possesses initially the state we want to teletransport, and the particle in which we want to put the state at the end of the process, are entangled by this operation. We have shown that by using two other atoms (ancillas), we can teleport the desired state. This is achieved using basically unitary one-atom operations and sweep operations; in particular, only two sweeps of the lattice of a third atomic level $|2\rangle$ are necessary. The process is fully realized via unitary transformations, and no measurement is performed; therefore, the process is completely reversible.

Let us make some remarks concerning other aspects of the suggested scheme. The state $|\phi\rangle$ can be initialised in the site 1 following a procedure similar to that which can be employed in the reading step of the teleportation scheme: (i) We consider an ancilla $A$ sufficiently separated from the rest of the atoms, and shine with a laser in such a way that a state $|\phi\rangle_{A}=a|0\rangle_{A}+b|2\rangle_{A}$ is created; (ii) then, we perform a Hadamard transform in the site 1 [18], and perform a sweep operation between $A$ and 1; (iii) a Hadamard transform is performed in the ancilla, and a second sweep operation between $A$ and 1 is performed. The result is that the ancilla becomes $|0\rangle_{A}$, while the site 1 acquires the state $|\phi\rangle_{1}$ as desired. In a similar way, we can put the final state of the site $N$ into a sufficiently isolated ancilla and perform a fluorescence experiment by shining with a laser. This allows us to read the final state, showing that the teleportation has been actually produced.

We want to emphasize that the purpose of this paper was to explain a possible implementation of a reversible teleportation scheme in an optical lattice, and not to show the most simple way to transport state $|\phi\rangle$ from one lattice site to another. This could have been achieved more easily by swapping the state of the first site with the ancilla as described in the paragraph above, and then the state of the ancilla with the second site. The difference between our scheme and simple swapping is that in our case the information is never transported to and localised in the ancilla, but spread over the total state and then localised in the second site, therefore exhibiting a non-trivial quantum operation.

Finally, we have used two ancillas, due to the fact that the ancillas are two-level atoms, and therefore cannot store four different values as required in the teleportation scheme. The teleportation can be also performed with just one ancilla, but the scheme becomes more complicated. Basically what is needed is a three-step process: (i) in a first step the ancilla reads the even sites of the lattice (as shown in Sec. V), and after that it is brought to $N$; after this step the possible states of the site $N$ become two instead of four; (ii) The reading process is repeated, bringing the ancilla to its original state $|0\rangle$; (iii) then, the ancilla reads the odd states as in Sec. V, and it is brought to $N$; after this step the possible states of the site $N$ are reduced to just one, and therefore one just needs to perform a known unitary transformation to conclude the teleportation.

We hope that the presented teleportation protocol will motivate further efforts into the realization of simple quantum networks in optical lattices.

We are grateful to J. I. Cirac for introducing us to this subject, and to M. Lewenstein for fruithful discussions. This work was supported by Deutsche Forschungsgemeinschaft under grant SFB 407 and by the EU through the TMR network ERBXTCT96-0002.

[1] A. Einstein, B. Podolsky and N. Rosen, Phys. Rev. 47, 777(1935).

[2] J. S. Bell, Speakable and unspeakable in quantum mechanics, Cambridge University Press (1987).

[3] D. M. Greenberger, M. Horne and A. Zeilinger, in Bell's theorem, quantum theory, and conceptions of the Universe, ed. by M. Kafatos, Kluwer, Dordrecht (1989), p. 69. 
[4] A. K. Ekert and R. Josza, Rev. Mod. Phys. 68, 733 (1996).

[5] A. K. Ekert, Phys. Rev. Lett. 67, 661 (1991).

[6] C.H. Bennett, G. Brassard, C. Crépeau, R. Josza, A. Peres and W. K. Wootters, Phys. Rev. Lett. 70, 1895 (1993).

[7] D. Bouwmeester, J.-W. Pan, K. Mattle, M. Eibl, H. Weinfurter and A. Zeilinger, Nature 390, 575 (1997);

D. Boschi, S. Branca, F. De Martini, L. Hardy, and S. Popescu, Phys. Rev. Lett. 80, 1121 (1998).

[8] A Bell-measurement consists of a joint measurement on two particles, determining whether they are in one of the four Bell states, namely $\left|\phi^{ \pm}\right\rangle=(|0\rangle|0\rangle \pm|1\rangle|1\rangle) / \sqrt{2}$, $\left|\psi^{ \pm}\right\rangle=(|0\rangle|1\rangle \pm|1\rangle|0\rangle) / \sqrt{2}$. In the experiments performed up to now, only $\left|\psi^{+}\right\rangle$and $\left|\psi^{-}\right\rangle$can be identified, but not $\left|\phi^{ \pm}\right\rangle$, whose identification is only possible if the two particles are coupled by some interaction. Concerning the problem of Bell measurements see for example, N. Lütkenhaus, J. Calsamiglia and K-A. Suominen, quant$\mathrm{ph} / 9809063$.

[9] S. L. Braunstein, Phys. Rev. A 53, 1900 (1996).

[10] M. A. Nielsen and C. M. Caves, Phys. Rev. A, 55, 2547 (1997).

[11] Q. A. Turchette, C. J. Hood, W. Lange, H. Mabuchi and H. J. Kimble, Phys. Rev. Lett. 75, 4510 (1995).

[12] A. Aspect, J. Dalibard and G. Roger, Phys. Rev. Lett. 49, 91 (1982).

[13] J. I. Cirac and P. Zoller, Phys. Rev. Lett. 74, 4091 (1995).

[14] D. Jaksch, H. J. Briegel, J. I. Cirac, C. W. Gardiner nad P. Zoller, Phys. Rev. Lett. 82, 1975 (1999).

[15] H. J. Briegel, T. Calarco, D. Jaksch, J. I. Cirac and P. Zoller, quant-ph/9904010.

[16] P. Shor, Phys. Rev. A 52, R2493 (1995).

[17] D. Jaksch, C. Bruder, J. I. Cirac, C. W. Gardiner and P. Zoller, Phys. Rev. Lett. 81, 3108 (1998).

[18] These one-atom operations can be achieved by shining a laser on the desired atom. However, it is not realistic to assume that only one atom is then affected, because the atoms are typically separated by a $\lambda / 2$ distance, where $\lambda$ is the wavelength of the laser which creates the lattice. Fortunately, our teleportation scheme does not employ one-atom unitary operations specifically for one site, but operations which can in principle be applied simultaneously to all the atoms of the lattice. The only exception is the ancilla, which is assumed to be placed sufficiently far away from the other atoms of the lattice, and therefore individual unitary operations can be performed on this particular atom.

[19] In principle, kinetic phases appear as well, due to the movement of the atoms, but these are trivial one-particle phases, which can always be incorporated into the definition of states $|0\rangle$ and $|1\rangle$.

[20] In principle, $|2\rangle_{A}|0\rangle_{j} \rightarrow e^{i \phi_{0}}|2\rangle_{A}|0\rangle_{j}$, whereas $|2\rangle_{A}|0\rangle_{j} \rightarrow$ $e^{i\left(\phi_{0}+\phi\right)}|2\rangle_{A}|1\rangle_{j}$, but as the phase $\phi_{0}$ appears anyway, it can be reabsorbed in the definition of $|2\rangle_{A}$, as it is also done with the kinetic phases 19. By setting $\phi=\pi$, we retrieve expression (16).

[21] As pointed out in Ref. 15], another, perhaps more elegant, way to solve this problem is to consider a three dimensional lattice, in which the ancilla can be vertically displaced upwards, moved, and displaced downwards to the desired site of the lattice.

\section{APPENDIX A: PROOF OF EQUATION (26)}

In this appendix we prove that after applying on the initial state (25) a Hadamard transform in all the $N$ sites (in the following we denote this operation as $H^{\oplus N}$ ), performing a shift operation (in the following we call it $L$ ), and applying again $H^{\oplus N}$, the state of the system becomes that of expression (26). We are proving this using induction arguments. Let us call $\mathcal{Q}_{N}=H^{\oplus(N)} L H^{\oplus(N)}$. It is easy to observe that for the case of 4 sites, expression (26) is fulfilled. Let us assume that for the case of $N=2 m$ sites, (26) is fulfilled, i.e.

$$
|\Phi\rangle_{1, \cdots, N}=\frac{1}{2^{m}} \sum_{J=0}^{2^{N}-1} c_{J}\left|\tilde{\psi}^{(J)}\right\rangle_{1 \cdots N-1} \tilde{U}_{N}^{(J) \dagger}|\phi\rangle_{N} .
$$

with $c_{J}= \pm 1$. Now, we are going to add two more sites $(N+1),(N+2)$ at the right of the site $N$. The effect of $\mathcal{Q}_{N+2}$ on the initial state for the $N+2$ sites, can be easily calculated from the state $|\Phi\rangle_{1, \cdots, N}$ using the unitary character of the operations, then:

$$
\begin{aligned}
& |\Phi\rangle_{1, \cdots, N+2}= \\
& \mathcal{Q}_{N+2}\left[\mathcal{Q}_{N}^{-1}|\Phi\rangle_{1, \cdots, N} \otimes|0\rangle_{N+1}|0\rangle_{N+2}\right] \\
& =\frac{1}{2^{m+1}} \sum_{J=0}^{2^{N}-1} c_{J}\left|\tilde{\psi}^{(J)}\right\rangle_{1 \cdots N-1} \otimes \\
& \sum_{k=0}^{3} b_{k}|k \operatorname{div} 2\rangle_{N}|k \bmod 2\rangle_{N+1} O^{(k)} \tilde{U}_{N}^{(J) \dagger}|\phi\rangle_{N+2} \\
& =\frac{1}{2^{m+1}} \sum_{J=0}^{2^{N+2}-1} c_{J}^{\prime}\left|\tilde{\psi}^{(J)}\right\rangle_{1 \cdots N+1} \tilde{U}_{N+2}^{(J) \dagger}|\phi\rangle_{N},
\end{aligned}
$$

where $O^{(0)} \equiv-i \sigma_{2}, O^{(1)} \equiv \sigma_{1}, O^{(2)} \equiv \sigma_{3}, O^{(4)} \equiv \mathbb{1}$, and $b_{k}$ and $c_{J}^{\prime}$ can be \pm 1 . Let us assume that $m$ is even, and therefore $\tilde{U}_{N}^{(J) \dagger}$ satisfies the requirements of Sec. $\mathrm{V}$. It is possible to obtain, after some calculation, that the new unitary operators $\tilde{U}_{N+2}^{\prime(J) \dagger}$ satisfy the same requirements but interchanging $S_{e, o}^{(J)} \bmod 2=0 \leftrightarrow S_{e, o}^{(J)} \bmod 2=1$. We note that the same applies if $m$ is odd. Therefore, we have proved that if for $N$ sites 26) is satisfied, this also holds for $N+2$ sites. Since for $N=4$ the statement is true, the proof is completed by induction. 